\title{
Bleaching induced tooth sensitivity: do the existing enamel craze lines increase sensitivity? A clinical study
}

Özcan, Mutlu ; Abdin, Sam ; Sipahi, Cumhur

\begin{abstract}
The aim of this clinical study was to evaluate whether or not an association exists between the presence of enamel craze lines and the prevalence of tooth sensitivity (TS) after in-office bleaching. Subjects that met the inclusion criteria $(\mathrm{N}=23)$ were screened to detect the existence of enamel craze lines. In total, 460 teeth were subjected to bleaching where $49 \%$ of them presented enamel craze lines. After bleaching (15\% hydrogen peroxide), the subjects were asked to rate the level of TS by answering a self-administered questionnaire. The majority of subjects $(91 \%)$ experienced TS at the first day of bleaching. The TS prevalence decreased gradually to $22 \%$ at second day, to $17 \%$ at third day, and to $9 \%$ at fourth day. After the fourth day, no subject reported TS. While $15 \%$ of teeth with craze lines presented TS, $11 \%$ of teeth with no craze lines also showed TS. A positive but weak correlation $(r=0.214)$ was found between the existence of enamel craze lines and TS. In this clinical study, higher incidence of TS was found with the use of $15 \%$ hydrogen peroxide bleaching agent compared to the previous studies. Patients who would undergo in-office bleaching should be informed that tooth sensitivity is a very often side effect but it may disappear within 1 week.
\end{abstract}

DOI: https://doi.org/10.1007/s10266-013-0104-7

Posted at the Zurich Open Repository and Archive, University of Zurich

ZORA URL: https://doi.org/10.5167/uzh-100653

Journal Article

Accepted Version

Originally published at:

Özcan, Mutlu; Abdin, Sam; Sipahi, Cumhur (2014). Bleaching induced tooth sensitivity: do the existing enamel craze lines increase sensitivity? A clinical study. Odontology / the Society of the Nippon Dental University, 102(2):197-202.

DOI: https://doi.org/10.1007/s10266-013-0104-7 


\section{Bleaching Induced Tooth Sensitivity: Do the Existing Enamel Craze Lines Increase Sensitivity? - A Clinical Study}

\section{Mutlu Özcan • Sam Abdin • Cumhur Sipahi}

Mutlu Özcan (®)

University of Zürich, Dental Materials Unit, Center for Dental and Oral Medicine Clinic for Fixed and Removable Prosthodontics and Dental Materials Science, Plattenstrasse 11, CH-8032, Zürich, Switzerland e-mail: mutluozcan@hotmail.com

\section{Sam Abdin}

University Medical Center Groningen, Department of Dentistry and Dental Hygiene, A. Deusinglaan 1, 9713 AV Groningen, The Netherlands

Cumhur Sipahi

Department of Prosthodontics, Center for Dental Sciences, Gulhane Military Medical Academy, 06018, Ankara, Turkey

Short title: Bleaching induced tooth sensitivity

\section{Corresponding to:}

Prof. Dr.med.dent. Mutlu Özcan, Ph.D University of Zürich Head of Dental Materials Unit Center for Dental and Oral Medicine Clinic for Fixed and Removable Prosthodontics and Dental Materials Science Plattenstrasse 11 $\mathrm{CH}-8032$, Zürich, Switzerland Tel: +41-44-634560 Fax: +41-44-6344305

e-mail: mutluozcan@hotmail.com 


\begin{abstract}
The aim of this clinical study was to evaluate whether or not an association exists between the presence of enamel craze lines and the prevalence of tooth sensitivity (TS) after in-office bleaching. Subjects that met the inclusion criteria $(\mathrm{N}=23)$ were screened to detect the existence of enamel craze lines. In total, 460 teeth were subjected to bleaching where $49 \%$ of them presented enamel craze lines. After bleaching (15\% hydrogen peroxide), the subjects were asked to rate the level of TS by answering a self-administered questionnaire. The majority of subjects (91\%) experienced TS at the first day of bleaching. The TS prevalence decreased gradually to $22 \%$ at second day, to $17 \%$ at third day, and to $9 \%$ at fourth day. After the fourth day, no subject reported TS. While $15 \%$ of teeth with craze lines presented TS, $11 \%$ of teeth with no craze lines also showed TS. A positive but weak correlation $(r=0.214)$ was found between the existence of enamel craze lines and TS. In this clinical study, higher incidence of TS was found with the use of $15 \%$ hydrogen peroxide bleaching agent compared to previous studies. Patients who would undergo in-office bleaching should be informed that tooth sensitivity is a very often side effect but it may disappear within one week.
\end{abstract}

Keywords Cracked tooth syndrome $\bullet$ Enamel craze lines $\bullet$ Office bleaching $\bullet$ Tooth bleaching $\bullet$ Tooth sensitivity • Tooth whitening 


\section{Introduction}

Discolored teeth may affect self-esteem of individuals depending on the society they live in. The demand of individuals for tooth whitening existed for more than 100 years and since then significant efforts have been directed towards understanding the nature of tooth discoloration and devising methods to eliminate them $[1,2]$. Currently, several methods are available to restore discolored dentition. These include mechanical or chemical stain removal, restorations with composite or ceramic veneers or full coverage crowns. The clinical procedure to restore stained dentition depends upon variables such as the nature and intensity of stain, age of the patient, and the desired optical change [3]. Of these methods, veneers and full coverage crowns are considered most invasive because sound tooth structure is removed to create room for the restoration. The least invasive procedure is to remove discoloration from discolored dentition is bleaching using oxygenating chemicals such as hydrogen peroxide $\left(\mathrm{H}_{2} \mathrm{O}_{2}\right)$. This method does not entail removal of sound tooth structure. Vital teeth can be bleached either chair side (in-office) by the dentist or through the application of bleaching agent in a mouth-guard by the patient [4,5], where the latter is usually referred to as doctor-prescribed nightguard vital bleaching or at-home bleaching. At-home whitening procedures are relatively inexpensive because they require less office visits and typically require the use of lower concentrations of peroxide $(+/-$ $10 \%)$ or carbamide peroxide $(10 \%)$. Sometimes it is difficult to achieve patient compliance with the mouthguards [4-6].

The in-office vital bleaching is commonly referred as power bleaching, where hydrogen peroxide solutions are used as bleaching agent activated by heat or light source. Previously, the in-office approach typically was conducted over a series of visits, utilizing high-concentration peroxides $(30-35 \%)$ under heat sources to increase the kinetics of stain removal [4-6]. Today, the in-office whitening method is developed to bleach teeth in a single office visit with a combination of a bleaching agent such as peroxide at lower concentrations and an auxiliary such as light. 
In both methods, carbamide peroxide can bleach the chromogens within the dentin, thereby reduce the body colour of the tooth. Such agents can be applied externally to the teeth (vital bleaching) or internally within the pulp chamber (non-vital bleaching) [7].

Clinical reports on the incidence of tooth sensitivity after vital bleaching procedures showed variations between studies [8-12]. At-home bleaching caused tooth sensitivity up to $78 \%$ in participants. With in-office bleaching procedures the incidence of tooth sensitivity was similar but the great majority of people who undergo the whitening procedures, were able to tolerate it well [9-12]. Yet, sensitivity was a critical problem for some participants. Schulte and Morisette [1] reported that sensitivity was severe enough to cause $14 \%$ of the participants to discontinue bleaching. Other studies reported a percentage of subjects who experienced sensitivity already from a specific source and not necessarily due to bleaching $[9,12]$. Haywood et al. [10] found that bleaching caused tooth sensitivity in $52 \%$ of the subjects and $31 \%$ of them experienced gingival sensitivity. Several studies showed that bleaching methods using only peroxides without light sources can cause tooth sensitivity, and this can be due to the used peroxide or one of the other ingredients in the bleaching gel [12-16]. On the other hand, it has been estimated that the incidence of tooth fractures or tooth cracks is to be 5 per 100 adults per year [17-20]. In fact, enamel surface cracks or craze lines, are relatively common in adult teeth [17-20]. However, there is sparse data in the literature about the association between the presence of enamel craze lines and the prevalence of tooth sensitivity during bleaching.

The objective of this clinical study therefore, was to investigate whether or not an association exists between the presence of enamel craze lines and the prevalence of tooth sensitivity after in-office bleaching. 


\section{Materials and methods}

\section{Patient selection}

The subjects were not admitted to the study if any of the following criteria were present: 1) younger than 18 years old or older than 55 years old, 2) not able to read and sign the informed consent document, 3) physically and psychologically not normal, 4) having general health-compromising conditions, 5) currently on medication, 6) used any medication within the past 14 days, 7) pregnant, 8) endodontic therapy, pulp pathosis, 9) any faulty restoration, 10) caries, 11) tooth wear, 12) gingival recession, 13) pre-existing tooth sensitivity on the teeth to be evaluated, 14) undergoing active periodontal and/or orthodontic therapy, 15) smokers, 16) residence outside the city, insufficient address for follow-up, or unwillingness to return for followup as outlined by the investigators.

In total, 23 healthy subjects (17 women and 6 men) could fulfill the inclusion criteria and be recruited to participate in the study from 62 interviewed subjects. The informed consents of the subjects were obtained before bleaching procedures. They were also informed that they would receive a questionnaire that they have to fill during the first week after the completion of bleaching procedures.

Tooth cracks, number, location and directions of enamel craze lines or any other enamel abnormalities were individually documented per tooth. Enamel craze lines were detected using indirect illumination. This was accomplished by placing a light source (XL3000, 3M ESPE, light output: $550 \mathrm{~mW} / \mathrm{cm}^{2}$ ) against the lingual surface of the teeth (Figs. 1a-b).

\section{Bleaching procedure}

A bleaching kit (Brite Smile Inc, 33431, Baco Raton, FL) containing peroxide gel, a light polymerizing isolation material, a hydrogel, a lip cream and a lip sunblock was used for bleaching procedures. The bleaching gel contained $15 \%$ hydrogen peroxide in a pH 6.5 hydrogel, glycerin and water to prevent tooth dehydration. 
The subjects were instructed to brush their teeth for 30 seconds. After tooth brushing, the initial tooth colors were determined using a color scale (Chromascop, Ivoclar Vivadent GmbH, D-73471, Ellwangen, Germany) for each subject. In order to ensure gingival protection, a brush-on isolation material (Opaldam) was applied along gingival margins in a width of approximately $1 \mathrm{~mm}$ in the treatment area before bleaching A cheek retractor was used to retract the labial comissurae and lips away from the treatment area. Cotton rolls were placed in bilateral vestibular sulci to isolate the treatment area from saliva. Bite blocks were used as jaw rests. A sun block and a hydration cream (Vaseline Petroleum Jelly) were applied onto the lips. The subjects wore an orange tinted protective eyewear during the bleaching procedure.

The bleaching gel was applied in a thickness of approximately $2 \mathrm{~mm}$ onto the buccal surfaces of all maxillary and mandibular anterior teeth using a strip. The incisors, canines and premolars were fully covered with the gel to ensure a uniform effect. Light was positioned according to the manufacturer's instructions using the integral bite appliance guide to set the distance between the teeth and the light source. All treatments lasted for 1 hour. Desiccation of the tooth surface was minimized by reapplication of hydroge every 20 minutes. The used light source was a stationary, short arc gas plasma lamp emitting light in bluegreen portion of the colour spectrum (400 - $505 \mathrm{~nm}$ ). The lamp simultaneously illuminated all maxillary and mandibular anterior teeth. After the completion of the bleaching procedures, the teeth were rinsed with air and water spraying for 1 minute. The final tooth colour was measured with the same colour scale.

\section{Tooth sensitivity test}

Directly after finishing the bleaching procedure, the subjects were interviewed and asked to fill the selfadministrated questionnaire for the documentation of any discomfort, pain or side effect that they experienced between the 1st and 7th day after the completion of bleaching treatment. They were also asked to document the location, level and the duration of the pain or sensitivity for up to 7 days after the bleaching procedure. Sensitivity was recorded using the following scale [10]: 
Grade 0: No sensitivity

Grade 1: Mild sensitivity (slight changes noted, no interference with function, well toleration)

Grade 2: Moderate sensitivity (definite changes noted, presence of functional interference, necessity of avoiding certain food)

Grade 3: Severe sensitivity (subject considered discontinuing the bleaching procedure, not able to tolerate the pain).

Statistical analysis

Data were analyzed from 3 sources namely, the patient questionnaires filled during seven days posttreatment, the results of the intake visit concerning the existence of enamel craze lines and the interview regarding possible experience of sensitivity held directly after finishing the bleaching procedure. To determine any correlation between the existence of enamel craze lines and tooth sensitivity after the in-office vital bleaching, the Pearson's Correlation test was performed using statistical software (SAS System for Windows: release 8.02/200; Cary, NC).

\section{Results}

All 23 subjects have completed the bleaching procedure. No one stopped the bleaching procedure for sensitivity or any other reason.

In total of 460 teeth were subjected to bleaching of which 226 (49\%) presented enamel craze lines. The numbers of craze lines in 20 treated teeth per patient ranged between 2 and 16 craze lines as maximum.

The incidence of tooth sensitivity ranged between 0 to 7 teeth per subject corresponding to 0 to $35 \%$ of the total treated teeth per subject (Table 1).

A total of $61(13 \%)$ of all bleached teeth experienced sensitivity at the 1 st treatment day. While a total of 35 $(15 \%)$ of the teeth with craze lines experienced sensitivity, $191(85 \%)$ of the teeth with craze lines showed nc 
sensitivity. The total number of teeth with no craze lines with sensitivity was 234 (51\%) whereas 26 (11\%) showed no craze lines but sensitivity (Fig. 2).

A positive but weak correlation was found between the incidence of enamel craze lines and tooth sensitivity ( $r=0.214)$ (Pearson's correlation).

The existence of sensitivity and the grade of the experienced sensitivity per day were as follows (Table 2):

$1^{\text {st }}$ day: While $78 \%$ of the studied population experienced mild tooth sensitivity (Grade 1 ), $13 \%$ experienced moderate sensitivity (Grade 2), 9\% experienced no sensitivity. Sensitivity level Grade 3 was not observed by any of the patients.

$2^{\text {nd }}$ day: $78 \%$ of the studied population experienced no sensitivity (Grade 0 ), $22 \%$ experienced mild tooth sensitivity (Grade 1) and no one experienced moderate or severe sensitivity (Grades 2 or 3).

$3^{\text {rd }}$ day: $83 \%$ of the studied population reported no sensitivity (Grade 0 ), $17 \%$ reported mild sensitivity (Grade 1). Similar to the previous day no one experienced moderate or severe sensitivity (Grades 2 or 3 ).

$4^{\text {th }}$ day: $91 \%$ of the studied population reported, no sensitivity (Grade 0), only $9 \%$ showed sensitivity Grade 1.

$5^{\text {th }}$ day: No one of the studied population ( $\left.0 \%\right)$ experienced any sensitivity.

The results of $6^{\text {th }}$ and $7^{\text {th }}$ day were similar to that of $5^{\text {th }}$ day, meaning that sensitivity disappeared completely.

\section{Discussion}

Craze lines are merely cracks in the enamel that do not extend to the dentin and are very common in adult teeth [17-19]. Despite the high incidence of microcracks seen in the teeth of adult populations, no specific study was found that evaluated the effect of these cracks on the diffusion of solutions such as peroxide intc the dentin and/or the pulp. The purpose of this clinical study was to evaluate the possible correlation between 
the existence of enamel craze lines and tooth sensitivity after an in-office vital bleaching procedure. The duration and rank of sensitivity was also of interest.

Previous in-vitro studies reported that peroxide could penetrate enamel and dentin, and enter the pulp chamber $[9,11,12]$. It was also stated that the amount of the peroxide detected in the pulp chamber was related to the concentration of the hydrogen peroxide in the preparations applied. Therefore, enamel craze lines could increase the diffusion rate of the peroxide gel into the dentin and consequently into the pulp, causing a higher incidence of tooth sensitivity.

In this clinical study, the incidence of tooth sensitivity in teeth with craze lines was evaluated and compared with those without craze lines. All teeth underwent the same bleaching procedure, using the same peroxide concentration for the same duration under the same light source. Therefore, a possible variable that could explain any differences in the incidence of tooth sensitivity was the increased diffusion of the peroxide gel intc the pulp due to the existence of enamel craze lines. Based on the results of this study, however a positive but weak correlation was found between the presence of enamel craze lines and tooth sensitivity after in-office vital bleaching.

The findings of the present study showed that sensitivity ranged predominantly from mild to moderate (Grade 1-2). No subject ceased the bleaching treatment because of severe sensitivity or any other side effects. After four days, none of the subjects experienced tooth sensitivity. These results were similar on day five, six and seven after the treatment. At the first day, $78 \%$ of the population experienced mild tooth sensitivity, $13 \%$ experienced moderate sensitivity and only $9 \%$ with no sensitivity. These results contradict with the results of the study performed by Rosenblum and Nathoo [5] who used the same bleaching material as the one used in this study (Brite Smile bleaching kit; $15 \% \mathrm{H}_{2} \mathrm{O}_{2}$ concentration), with the same application procedure. In their study, the subjects $(\mathrm{N}=75)$ were divided into 3 groups, 25 subjects each. The first group was bleached using $15 \% \mathrm{H}_{2} \mathrm{O}_{2}$ in combination with light. The subjects of the second group were bleached 
using only $15 \% \mathrm{H}_{2} \mathrm{O}_{2}$, and subjects of the third group received only placebo gel in combination with light. The incidence of sensitivity was $24 \%, 32 \%$ and $16 \%$, respectively. Subjects were directly interviewed after finishing the bleaching procedure, and were asked about the experienced sensitivity during the procedure. In our study, the subjects were asked to note the experienced sensitivity starting from day the bleaching procedure was performed ( $1^{\text {st }}$ day), and not only during the bleaching procedure. This could be one possible explanation of the discrepancy between the results of the two studies especially on the $1^{\text {st }}$ day. Since with and without the light source, tooth sensitivity was present, light source could not be considered as the main reason for sensitivity. It should also be noted that the study population was larger in that study.

In other clinical studies, tooth sensitivity was very common $[7-9,11,12]$. While the studies that evaluated sensitivity after night-guard vital bleaching reported that sensitivity ranging between 7 and $78 \%[7,9,11]$, after in-office vital bleaching, sensitivity was reported to be between 24 and $78 \%$ of the studied population $[11,12]$. Also, Cohen and Chase [8] reported the incidence of tooth sensitivity in $78 \%$ of the studied subjects, where a high concentration of peroxide gel $\left(30 \% \mathrm{H}_{2} \mathrm{O}_{2}\right)$ was used and the bleaching procedure was divided into 3 visits of 30 minutes each. None of these studies reported the existence of enamel craze lines or mentioned a possible relation to the experienced sensitivity.

The light source used in this study was a plasma arc lamp. In one study, temperature increase was noted in the pulp chamber during bleaching using light emitting diode (LED)/laser light source [21], and in another one, LED light was suggested to be safe for periodontal and pulp tissue but halogen light was advised to be used with care [22]. Future studies are warranted focusing on the factors such as enamel craze lines. diffusion parameters of $\mathrm{H}_{2} \mathrm{O}_{2}$ to the pulp in relation with the light source solely and in combination.

It can be concluded that there is no strong causal relation between the two variables. Thus it cannot be assured that the existence of enamel craze lines is in direct relation with tooth sensitivity, or that craze lines can increase the potential of a higher diffusion of the peroxide gel into the dentine and pulp chamber. This 
hypothesis has to be verified in larger populations or with in vitro studies evaluating the diffusion rate of peroxide in teeth with craze lines versus teeth with no craze lines.

\section{Conclusions}

In this clinical study, higher incidence of tooth sensitivity was found with the use of $15 \%$ hydrogen peroxide bleaching agent compared to previous studies. There seems to be a trend that enamel craze lines are potential factors in increasing tooth sensitivity after in-office vital bleaching but due to the weak correlation, this relation cannot be stated. Experienced tooth sensitivity after tooth bleaching with the bleaching material tested, is temporary and it lasts for only few days. Patients who would undergo office bleaching should be informed that tooth sensitivity is a very often side effect but it may disappear within one week. The results should be confirmed in larger populations.

\section{Conflict of Interest}

The authors declare that they have no conflict of interest. 


\section{References}

1. Setien VJ, Roshan S, Nelson PW. Clinical management of discolored teeth. Gen Dent. 2008;56:294-300.

2. Chhabra N, Singbal KP. Viable approach to manage superficial enamel discoloration. Contemp Clin Dent. 2010;1:284-7.

3. Kwon SR. Whitening the single discolored tooth. Dent Clin North Am. 2011;55:229-39.

4. Nathanson D, Parra C. Bleaching vital teeth: A review and clinical study. Compend Contin Educ Dent. $1987 ; 8: 490-7$.

5. Rosenblum MA, Nathoo SA. Clinical study to evaluate the safety and efficacy of the BriteSmile 2000 InOffice tooth whitening system. NJ Dental School, University of Medicine and Dentistry of New Jersey, University Heights, Newark, NJ 07103.

6. Tavares M, Stultz J, Newman M, Smith V, Kent R, Carpino E, Goodson JM. Light augments tooth whitening with peroxide. J Am Dent Assoc. 2003;134:167-75.

7. Tredwin CJ, Naik SN, Lewis J, Scully C. Hydrogen peroxide tooth-whitening (bleaching) products: Review of adverse effects and safety issues. Br Dent J. 2006;200:371-6.

8. Cohen SC, Chase C. Human pulpal response to bleaching procedures on vital teeth. J Endod. 1979;5:1348.

9. Howard WR. Patient-applied tooth whiteners, are they safe, effective with supervision? J Am Dent Assoc. 1992;123:57-60.

10. Haywood VB, Leonard RH, Nelson CF, Brunson WD. Effectiveness, side effects and long-term status of nightguard vital bleaching. J Am Dent Assoc. 1994;125:1219-26.

11. Schulte JR, Morrisette DB. The effects of bleaching application time on the dental pulp. J Am Dent Assoc. 1994;125:1330-5. 
12. Jorgensen M, Carroll W. Incidence of tooth sensitivity after home whitening treatment. J Am Dent Assoc. 2002;133:1076-82.

13. Bowles $\mathrm{WH}$, Ugwuneri Z, Pulp chamber penetration by hydrogen peroxide following vital bleaching procedure. J Endod. 1987;13:375-7.

14. Cooper J, Bokmeyer T, Bowles W. Penetration of the pulp chamber by carbamide peroxide bleaching agents. J Endod. 1992;18:315-7.

15. Gökay O, Tunçbilek M, Ertan R. Penetration of the pulp chamber by carbamide peroxide bleaching agents on teeth restored with a composite resin. J Oral Rehabil. 2000;27:428-31.

16. Dahl JE. Tooth Bleaching - a critical review of the biological aspects. Int Am Assoc Dent Res 2003;14(4):292-304.

17. Lynch C, Mc Connell R. The cracked tooth syndrome. J Can Dent Assoc. 2002;68:470-5.

18. Clark D, Sheets C, Paquette J. Definitive diagnosis of early enamel and dentin cracks based on microscopic evaluation. J Esthet Restor Dent. 2003;15:391-401.

19. Davich M. Clinical detection and decision-making considerations for cracked teeth. Endod Therapy. 2004;4:4-7.

20. Hewlett E. Etiology and management of whitening-induced tooth hypersensitivity. J Calif Dent Assoc. 2007;35:499-506.

21. Zamboni SC, Castanho GM, Torres CR, Nogueira L Jr, Borges AB, Bottino MA. Influence of the coloring agent concentration on bleaching gel and pulp chamber temperatures during dental bleaching. Gen Dent. 2010;58:e36-41.

22. Kabbach W, Zezell DM, Pereira TM, Albero FG, Clavijo VR, de Andrade MF. A thermal investigation of dental bleaching in vitro. Photomed Laser Surg. 2008;26:489-93. 
Figure legends:

Tables

Table 1 Total number and percentage (\%) of teeth with and without craze lines and related tooth sensitivity

Table 1 Incidence and severity of tooth sensitivity in grades per day; Grade 0: No sensitivity; Grade 1: Mild sensitivity (slight changes noted, no interference with function, well toleration); Grade 2: Moderate sensitivity (definite changes noted, presence of functional interference, necessity of avoiding certain food); Grade 3 : Severe sensitivity (subject considered discontinuing the bleaching procedure, not able to tolerate the pain)

\section{Figures}

Figs. 1a-b. Detection of enamel craze line or cracks using a) blue light transillumination, b) inspection light Fig. 2 Number of enamel craze lines and tooth sensitivity per patient 


\section{Tables:}

\begin{tabular}{|l|c|c|}
\hline \multicolumn{1}{|c|}{ Teeth and sensitivity conditions } & N & \% \\
\hline Teeth with craze lines & 226 & 49 \\
\hline Teeth with no craze lines & 234 & 51 \\
\hline With craze lines with sensitivity & 35 & 15 \\
\hline With craze lines and no sensitivity & 191 & 85 \\
\hline No craze lines with sensitivity & 26 & 11 \\
\hline No craze lines and no sensitivity & 208 & 89 \\
\hline
\end{tabular}

Table 1 Total number and percentage (\%) of teeth with and without craze lines and related tooth sensitivity 


\begin{tabular}{|c|c|c|c|}
\hline \multicolumn{4}{|c|}{ Incidence and Severity of Tooth Sensitivity } \\
\hline \multirow{6}{*}{$\begin{array}{l}\text { 1st } \\
\text { Day }\end{array}$} & Grade & $\mathbf{N}$ & $\%$ \\
\hline & 0 & 2 & 9 \\
\hline & 1 & 18 & 78 \\
\hline & 2 & 3 & 13 \\
\hline & 3 & 0 & 0 \\
\hline & Total & 23 & 100 \\
\hline & & & \\
\hline \multirow{6}{*}{$\begin{array}{l}\text { 2nd } \\
\text { Day }\end{array}$} & Grade & $\mathbf{N}$ & $\%$ \\
\hline & 0 & 18 & 78 \\
\hline & 1 & 5 & 22 \\
\hline & 2 & 0 & 0 \\
\hline & 3 & 0 & 0 \\
\hline & Total & 23 & 100 \\
\hline \multirow{6}{*}{$\begin{array}{l}\text { 3rd } \\
\text { Day }\end{array}$} & Grade & $\mathbf{N}$ & $\%$ \\
\hline & 0 & 19 & 83 \\
\hline & 1 & 4 & 17 \\
\hline & 2 & 0 & 0 \\
\hline & 3 & 0 & 0 \\
\hline & Total & 23 & 100 \\
\hline & & & \\
\hline \multirow{6}{*}{$\begin{array}{l}\text { 4th } \\
\text { Day }\end{array}$} & Grade & $\mathbf{N}$ & $\%$ \\
\hline & 0 & 21 & 91 \\
\hline & 1 & 2 & 9 \\
\hline & 2 & 0 & 0 \\
\hline & 3 & 0 & 0 \\
\hline & Total & 23 & 100 \\
\hline & & & \\
\hline \multirow{5}{*}{$\begin{array}{l}\text { 5th } \\
\text { Day }\end{array}$} & $\frac{\text { Grade }}{0}$ & $\frac{N}{23}$ & $\frac{\%}{100}$ \\
\hline & 1 & 0 & 0 \\
\hline & 2 & 0 & 0 \\
\hline & 3 & 0 & 0 \\
\hline & Total & 23 & 100 \\
\hline
\end{tabular}

Table 1 Incidence and severity of tooth sensitivity in grades per day; Grade 0: No sensitivity; Grade 1: Mild sensitivity (slight changes noted, no interference with function, well toleration); Grade 2: Moderate sensitivity (definite changes noted, presence of functional interference, necessity of avoiding certain food); Grade 3: Severe sensitivity (subject considered discontinuing the bleaching procedure, not able to tolerate the pain) 


\section{Figures:}
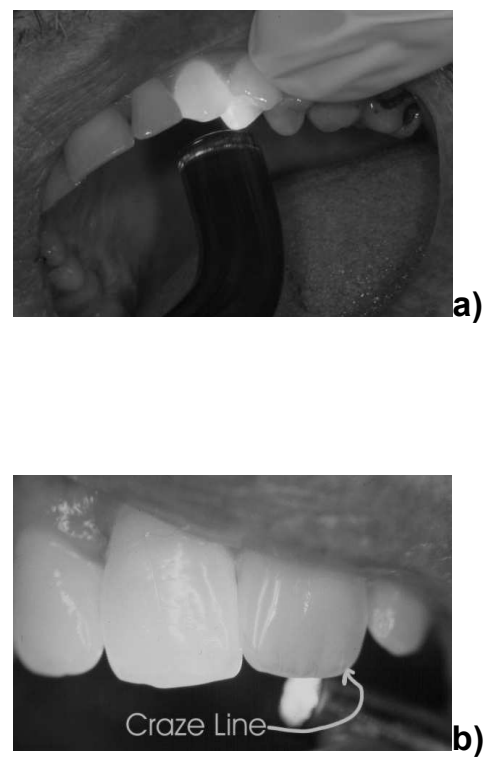

Figs. 1a-b. Detection of enamel craze line or cracks using a) blue light transillumination, b) inspection light

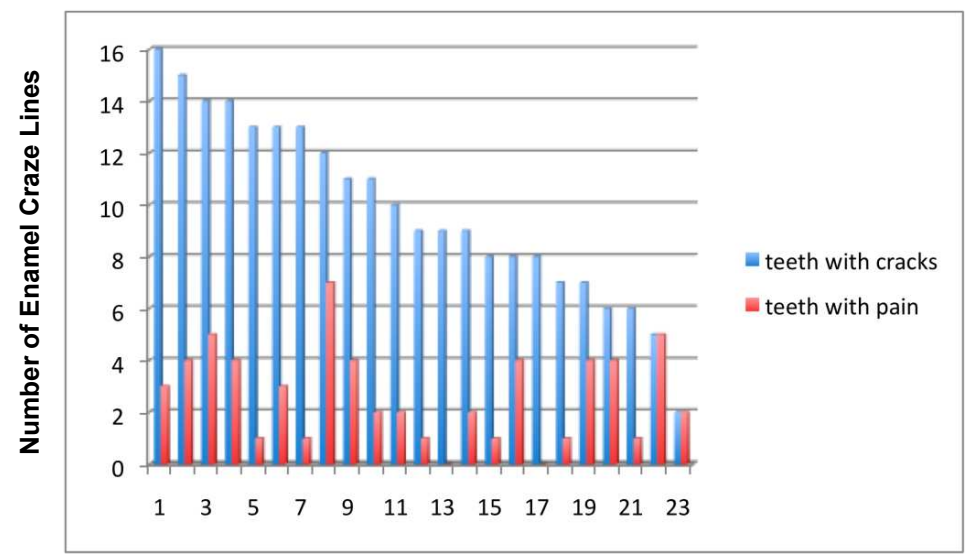

Patients

Fig. 2 Number of enamel craze lines and tooth sensitivity per patient 